\title{
An Analysis of Four-quark Energies in SU(2) Lattice Monte Carlo using the Flux-tube Symmetry
}

\author{
S. Furui $⿴ 囗 ⿱ 一 一)$ \\ School of Science and Engineering, \\ Teikyo University, Utsunomiya 320, Japan \\ A.M.Green 团and B.Masud \\ Research Institute for Theoretical Physics, \\ P.O.Box 9 SF-00014 University of Helsinki, Finland
}

November 15, 2018

\begin{abstract}
Energies of four-quark systems calculated by the static quenched $\mathrm{SU}(2)$ lattice Monte Carlo method are analyzed in $2 \times 2$ bases for square, rectangle, tilted rectangle, linear and quadrilateral geometry configurations and in $3 \times 3$ bases for a non-planar geometry configuration. For small interquark distances, a lattice effect is taken into account by considering perimeter dependent terms which are characterized by the cubic symmetry. It is then found that a parameter $f$ - that can be identified as a gluon field overlap factor - is rather well described by the form $\exp \left(-\left[b_{s} E \mathcal{A}+\sqrt{b_{s}} F \mathcal{P}\right]\right)$, where $\mathcal{A}$ and $\mathcal{P}$ are the area and perimeter mainly defined by the positions of the four quarks, $b_{s}$ is the string constant in the 2 -quark potentials and $E, F$ are constants.
\end{abstract}

*Supported by JSPS and Academy of Finland. e-mail furui@dream.ics. teikyo-u.ac.jp $\dagger$ †e-mail green@phcu.helsinki.fi

${ }_{\ddagger}^{\ddagger}$ present address:Department of Physics, Punjab University, Lahore, Pakistan 


\section{Introduction}

QCD is thought to be the basic theory describing the interaction between quarks and gluons. Unfortunately, until now, because of numerical reasons, this can only be checked in systems containing 2 or 3 quarks. Therefore to make the theory more useful, methods are needed that are much less numerical intensive than lattice Monte Carlo. These methods should agree with the Monte Carlo approach for the few quark systems - the assumption being that their natural extension to multiquark systems would also give correct results. Since many of the multiquark systems of interest are derived from hadronhadron scattering, in which different quark clusters scatter and rearrange, it is the $q^{2} \bar{q}^{2}$ system that must be first understood. Therefore, in several attempts to describe meson-meson scatterings from QCD, the resonating group method in which a meson is treated as a $q \bar{q}$ cluster is a promising approach. In $\mathrm{I}=2 \pi \pi$ and $\mathrm{I}=1 K \bar{K}$ scattering the amplitude is dominated by the direct gluon and quark exchange, and the quark-pair annihilation is expected to play a minor role. Therefore, as a first step, the detailed analysis of quark exchange processes from first principles or from lattice QCD is desirable. In a series of papers on the four-quark energies in $\mathrm{SU}(2)$ lattice Monte Carlo[1, 2], we considered configurations of two static $q \bar{q}$ clusters, which we called basis A and the quark interchanged two $q \bar{q}$ state basis B. We then analyzed the energy spectra in a framework similar to the resonating group method using the corresponding $2 \times 2$ bases. It was observed that the transition potential $v_{A B}$ connecting the bases needed to be multiplied by a factor $f$ - that can be interpreted as a gluon field overlap factor - in order that the solution of the matrix equation in this model coincided with the two lowest eigen energies of the Monte Carlo calculation.

Recently the configurations of the four-quark system were extended to include large square(LS), rectangular(R), tilted rectangle(TR), linear(L), quadrilateral (Q) and nonplanar(NP) geometries [3]. An analysis similar to the [1, 2 showed that, except for the NP case, the gluon overlap factor $f_{0}$ derived from the ground state energy and the $f_{1}$ derived from the first excited state are close to each other, but not identical. In the NP case, the agreement was worse.

For simplicity of the model, we expect theoretically $f_{0}=f_{1}$ and we tenta-

tively attribute the small difference in $f_{0}$ and $f_{1}$ as numerical uncertainties of the Monte Carlo calculation. In the NP case, however, the analysis in terms 
of $2 \times 2$ bases could, itself, be an origin of the difficulty.

In the $\mathrm{SU}(2)$ lattice Monte Carlo approach, the transition between different configurations occurs through multiplications of plaquettes, which characterize the gluon interaction, with the bases wave function. In the NP case, some plaquettes are 3-dimensional while in the other five cases they are purely 2-dimensional. A calculation of the glueball spectrum in lattice Monte Carlo shows that the 3-dimensional bent plaquette contains an irreducible representation of the cubic group which violates the space parity. In our case gluon configurations are pinned down by the position of the quarks and anti-quarks and the results of the glueball calculation cannot be applied directly, but evenso we expect that the symmetry of the plaquettes, which makes the transition from basis A to B could play an important role.

The factor $f$ comes from the flux-tube rearrangement. In the case of TR, Q and NP, the flux tubes of basis B are not straight lines on the underlying lattice but they are a superposition of the shortest paths along the links. The cubic symmetry of the plaquettes that transform the basis A to the basis B of $\mathrm{TR}$ and $\mathrm{Q}$ have different irreducible representations of the cubic group, but in the NP case, there are two plaquettes that belong to the same irreducible representation. The excited states of the NP case could be the hybrids i.e. $q \bar{q}$ with gluonic excitations. We, therefore, consider two types of hybrids $B^{+}$ and $B^{-}$and analyze the NP data in a $3 \times 3$ basis using $\mathrm{A}$ and $B^{ \pm}$.

In order to extract parameters for use in a resonating group calculation, the continuum limit, in which the cubic symmetry is replaced by the rotation symmetry, is to be considered. Thus, parameters which are specific to the cubic symmetry are not desirable. We, therefore, parametrize the factor $f$ by a superposition of an area dependent term and a perimeter dependent term. When the area is not a plane surface, the area that we choose is not the sum of the area of the plaquettes but an approximate minimal surface that is bounded by the links. The perimeter is kept to be the length of links on the plaquette, since we want to respect the cubic symmetry which is essential for the parametrization. We check whether the parameters for different configurations like LS and $\mathrm{R}$ have a common parameter when the two configurations possess the same cubic symmetry. The success or failure of this approach is given by the degree to which the various configurations are described by the same parameters. Since the gluon overlap factor is not a physical quantity, it could depend on the lattice size. We expect that the perimeter dependence decreases as the lattice size becomes larger and in the 
continuum limit the area term dominates.

In sect. 2 we present the model for fitting the data of the lattice Monte Carlo, and the numerical results are shown in sect.3. Finally in sect.4 some conclusions are drawn.

\section{Model}

In fitting the lattice Monte Carlo we could choose three model state bases [1, [2].

- A: Two links of equal length d that connect $q \bar{q}$ on 1-3 and on 2-4.

- B: Two links that connect $q \bar{q}$ on 1-4 and on 2-3, such that the number of bending points is a minimum.

- C: Two links that connect between $q q$ and $\bar{q} \bar{q}$ on $1-2$ and $3-4$, such that the number of bending points is the minimum. This basis can be regarded as a baryon anti-baryon system in $\mathrm{SU}(2)$.

As explained in the Appendix of [3] the three states are constructed by contracting the operator

$$
T_{i j k l}=P_{i r}\left(z, x_{1}\right) q_{r}\left(x_{1}\right) P_{j s}\left(z, x_{2}\right) q_{s}\left(x_{2}\right) P_{k t}\left(z, x_{3}\right) q_{t}\left(x_{3}\right) P_{l u}\left(z, x_{4}\right) q_{u}\left(x_{4}\right) \mid 0>
$$

with tensors

$$
A_{i j k l}=\epsilon_{i j} \epsilon_{k l}, \quad B_{i j k l}=\epsilon_{i l} \epsilon_{k j} \quad \text { and } \quad C_{i j k l}=\epsilon_{i k} \epsilon_{j l} .
$$

Here, in $\mathrm{SU}(2)$, the quark field is described by $q_{i}(x)$ and the antiquark field by $\overline{q_{i}(x)}=-\epsilon_{i j} q_{j}(x)$ and $P_{i j}(z, x)$ is the colour flux path from $\mathrm{z}$ to $\mathrm{x}$. Due to the equation $\epsilon_{i k} \epsilon_{j l}=\delta_{i j} \delta_{k l}-\delta_{i l} \delta_{k j}$, the base $\mathrm{C}$ is equal to $\mathrm{A}-\mathrm{B}$ if $\mathrm{z}$ can be chosen to be a common point of the paths of $\mathrm{B}$. This is the case for linear $(\mathrm{L})$, base $\underline{B_{2}}$ of quadrilateral(Q) and base $\underline{B_{1}}, \underline{B_{2}}$ and $\underline{B_{3}}$ of non-planar(NP)(see figs. 4,5 and 6$)$. If there is not a common point, $P_{k l}(z, x)$ for $\mathrm{B}$ as well as for A should contain a product of links that starts from $\mathrm{z}$ and goes to a quark in $\mathrm{B}$ and returns to $\mathrm{z}$, and that starts from $\mathrm{z}$ and goes to a quark in $\mathrm{A}$ and returns to $\mathrm{z}$, respectively. A difference in this expectation value for $\mathrm{A}$ and $\mathrm{B}$ would make $\mathrm{C}$ different from $\mathrm{A}-\mathrm{B}$, but numerical calculation indicates 
that $\mathrm{C}$ is approximately expressed as a linear combination of $\mathrm{A}$ and $\mathrm{B}$, since combinations $\mathrm{A}+\mathrm{B}, \mathrm{A}+\mathrm{C}, \mathrm{B}+\mathrm{C}$ give very similar energy spectra 3 . In our colour SU(2) static model, we assign two meson states to $\mathrm{A}$ and $\mathrm{B}$, and a baryon anti-baryon state to $\mathrm{C}$. Therefore, base $\mathrm{C}$ is regarded as redundant and we extract the effective potential in the space $\mathrm{A}$, in the space $\mathrm{B}$ and the transition potential between $\mathrm{A}$ and $\mathrm{B}$ from the lattice numerical data.

In the actual calculation the links are fuzzed [4]. Here, fuzzing means replacing a link $U_{i j}$ by a linear combination of $U_{i j}=e^{i g_{0} A_{\mu} a}$ and a staple $U_{i k} U_{k l} U_{l j}=e^{i g_{0} A_{\nu} a} e^{i g_{0} A_{\mu} a} e^{-i g_{0} A_{\nu} a}$, where $\mu \neq \pm \nu$ and $\nu \neq 4$. It enhances the overlap of the configuration and has the same effect as tadpole renormalization of the gluon mass.

In the strong coupling approximation of $\mathrm{SU}(2)$ gauge theory, the Wilson matrix for a $q \bar{q}$ system is

$$
<W\left(L, T, g^{2}\right)>\simeq\left(\frac{1}{2 g^{2}}\right)^{L T / a^{2}}=\exp \left(-b_{s} L T\right),
$$

where $\mathrm{L}$ is the length of the link between $q$ and $\bar{q}, \mathrm{~T}$ is the Euclidean time, $b_{s}$ is the string tension and $a$ is the lattice constant.

A generalization to the $q \bar{q} q \bar{q}$ system, where the $q$ and $\bar{q}$ are on the alternate corners of a rectangle of sides $L_{1}$ and $L_{2}$, is [5, 6]

$$
W=\left[\begin{array}{cc}
e^{-2 b_{s} L_{1} T} & \epsilon \\
\epsilon & e^{-2 b_{s} L_{2} T}
\end{array}\right]
$$

where $\epsilon$ is a sum over transitions at time t:

$$
\epsilon=-\sum_{t} e^{-2 b_{s} L_{2}(T-t)} e^{-b_{s} L_{1} L_{2}} e^{-2 b_{s} L_{1} t} .
$$

In Hilbert space the transfer matrix $\mathrm{T}$, which satisfies

$$
\begin{gathered}
Z=\int(d U) e^{-S}=\operatorname{Tr} T^{N} \\
S=-\frac{2 a}{g_{t}^{2} a_{0}} \sum_{i j \text { (spatial) }} \operatorname{Re} \operatorname{Tr}\left(U_{i j, t+1}^{-1} U_{i j, t}\right)-\frac{2 a_{0}}{g_{s}^{2} a} \sum_{p, t} \operatorname{Re} \operatorname{Tr}\left(U(p)_{t}\right)
\end{gathered}
$$

has the matrix element - see ref.[7] page 103 -

$$
<U^{\prime}|T| U>=\exp \left(\frac{2 a}{g_{t}^{2} a_{0}} \sum_{i j} \operatorname{Re} \operatorname{Tr}\left(U_{i j}^{\prime}-1 U_{i j}\right)\right) \times \exp \left(\frac{2 a_{0}}{g_{s}^{2} a} \sum_{p} \operatorname{Re} \operatorname{Tr}(U(p))\right),
$$


where $p$ means a plaquette, $a_{0}$ is the time direction lattice spacing and $a$ is the space direction lattice spacing. The bare coupling constants $g_{s}$ and $g_{t}$ agree to the lowest order $g_{s}^{2}=g_{t}^{2}+O\left(g_{t}^{4}\right)$.

By a group operation g, $U_{i j}$ transforms to $U_{i j}^{\prime}=g U_{i j}$ and we define unitary operators $R_{i j}(g)|U>=| U^{\prime}>$. In terms of these quantities, $\mathrm{T}$ takes the form

$$
T=\prod_{i j}\left(\sum_{g} R_{i j}(g) \exp \left(\frac{2 a}{g_{t}^{2} a_{0}} \operatorname{Re} \operatorname{Tr} g\right)\right) \times \exp \left(\frac{2 a_{0}}{g_{s}^{2} a} \sum_{p} \operatorname{Re} \operatorname{Tr} U(p)\right) .
$$

The area term $\exp \left(-b_{s} L_{1} L_{2}\right)$ in eq.(4) originates from the sum of the spatial plaquettes as they tile the large rectangular plaquette of $L_{1} \times L_{2}$. Therefore, the dominant part of the transition potential between the model space $\mathrm{A}$ and the model space $\mathrm{B}$ is written in the form $v_{A B} \exp \left(-b_{s} E \mathcal{A}\right)$ where $\mathcal{A}$ is the area of the plaquette that allows transitions between the bases $\mathrm{A}$ and $\mathrm{B}$, and $E$ is to be determined by fitting the Monte Carlo lattice data.

When the plaquette passes through 2 quarks and 2 antiquarks then the degeneracy of the colour configurations or the coherence of the products of $U_{i j}$ along the perimeter becomes important. The gauge transformation $g$ that connects between the degenerate $Z_{2}$ bases which are eigen states of the Cartan subalgebra $t^{a} \theta^{a}$, where for $\mathrm{SU}(2) t^{a}=i \sigma^{a} / 2$ and $t^{a} \theta_{\mu}^{a}$ is proportional to $i \sigma^{3} / 2 \theta$, would be expressed as a product of the form $\exp \left(t^{a} \theta_{\mu}^{a} x_{\mu}\right)$ which is proportional to the exponential of the length of the perimeter. Thus, in addition to the product of the spatial tiles, which can be obtained by taking $g=1$, we take into account the transition between different $Z_{2}$ bases which are proportional to the length of the perimeter.

Incorporation of the perimeter term via renormalization e.g. via the self energy given by the tadpole diagram is discussed also in the large $\mathrm{N}$ expansion [10]. Therefore, we assume that the potential containing this correction is given in a form $v_{A B} \exp \left(-\left[b_{s} E \mathcal{A}+\sqrt{b_{s}} A_{n} \mathcal{P}\right]\right)$ where $\mathcal{P}$ is the length of the perimeter and $\sqrt{b}_{s}$ is included to make $A_{n}$ dimensionless. This form has been derived from the case where the four quarks are at the corners of a rectangle. For this the area $\mathcal{A}$ and the perimeter $\mathcal{P}$ have clear definitions. The further assumption is now made that, in general, $\mathcal{A}$ is the natural continuum values - independent of the underlying lattice structure. This is similar to describing the two-quark potential on the lattice as $V(r)=-e / r+b_{s} r+v_{0}$, where $r$ is the shortest distance between the two quarks. It is known that 
this form is good for $r \geq 2 a$. However, for smaller values of $r$ the lattized form

$$
V\left(r_{i j}\right)=-\left(e / r_{i j}\right)_{L}+b_{s} r+v_{0}
$$

is neccessary - see ref. [2

For the same reasons, those four-quark configurations, where the quarks are within two lattice spacings, probably contain effects arising from the underlying lattice. We keep the lattice structure for the perimeter term $\mathcal{P}$ and take into account this effect partially.

\subsection{Irreducible representations of the cubic group}

We consider now g as an element of the cubic group or the crystallographic point group. It has the effect of rotating links on a plaquette and we are interested in group operations which connect a product of $U_{i j}$ to a product of $U_{i j}^{\prime}$ which differ only by a cyclic permutation of the links. The Wilson loop at a fixed time $\tau$, or the time-local operator, is characterized by Ltuples $\mathcal{O}_{m}(p)=\left(\hat{f}_{1}, \cdots, \hat{f}_{L}\right)$ and a class formed by all the L-tuples, which are identical up to cyclic permutation, is denoted by $\left[\hat{f}_{1}, \cdots, \hat{f}_{L}\right]$. The suffix $m$ specifies the orientation of the plaquette and this numbering follows the definition of 80 .

Here the $\hat{f}_{j}$ 's stand for unit vectors in the $\mathrm{x}-, \mathrm{y}$ - and $\mathrm{z}$ - directions: $e_{1}, e_{2}$ and $e_{3}[\mathbb{8}]$. The charge conjugation transforms $C\left[\hat{f}_{1}, \cdots, \hat{f}_{L}\right]=\left[-\hat{f}_{L}, \cdots,-\hat{f}_{1}\right]$. We define $\mathcal{O}_{m}^{r}(p)=\left(\hat{f}_{L}, \cdots, \hat{f}_{1}\right)$. In usual $\mathrm{SU}(\mathrm{N}), \mathrm{C}$-parity positive and negative bases are given by $\left[\hat{f}_{1}, \cdots, \hat{f}_{L}\right] \pm\left[-\hat{f}_{L}, \cdots,-\hat{f}_{1}\right]$. In the case of $\mathrm{SU}(2)$, there are only C-parity positive states as the bases.

On the lattice the bases are transformed by the proper rotations $\mathcal{M}_{g}$ of the crystallographic point group which are divided into five classes [9];

1. $E$ (identity)

2. $C_{3}=\left\{C_{3 h}, C_{3 h}^{-1}\right\}(\mathrm{h}=\alpha, \beta, \gamma, \delta)$

3. $C_{4}^{2}=\left\{C_{4 h}^{2}\right\}(\mathrm{h}=\mathrm{x}, \mathrm{y}, \mathrm{z})$

4. $C_{4}=\left\{C_{4 h}, C_{4 h}^{-1}\right\}(\mathrm{h}=\mathrm{x}, \mathrm{y}, \mathrm{z})$

5. $C_{2}=\left\{C_{2 h}\right\}(\mathrm{h}=\mathrm{a}, \mathrm{b}, \mathrm{c}, \mathrm{d}, \mathrm{e}, \mathrm{f})$

The operations on the bases are given by $\mathcal{M}_{g}\left[\hat{f}_{1}, \cdots, \hat{f}_{L}\right]=\left[M_{g} \hat{f}_{1}, \cdots, M_{g} \hat{f}_{L}\right]$. The main difference of the classification of the type of the plaquettes here as 
compared to other lattice calculations of the glueball spectrum [8] is that we fix the configuration of the links in our model space and so we restrict $M_{g}$ such that $\left[M_{g} \hat{f}_{1}, \cdots, M_{g} \hat{f}_{L}\right]$ belongs to the class of $\left[\hat{f}_{1}, \cdots, \hat{f}_{L}\right]+\left[-\hat{f}_{L}, \cdots,-\hat{f}_{1}\right]$. Actually, the link between $q$ and $\bar{q}$ is fuzzed and we do not know the exact overlap factor between the states obtained by multiplying a plaquette and plaquettes properly rotated which are specified by $C_{k}$. We parametrize the effect of the overlap of the links on the perimeter of the plaquette by a correction to the above area law dependent term and search the parameters by fitting the data.

We specify the plaquettes that allow transitions between the spacial wave function of A into B in the large square(LS), rectangle( $\mathrm{R})$, tilted rectangle(TR), linear(L), quadrilateral $(\mathrm{Q})$ and non-planar(NP) geometries as follows.

\section{LS}

The bases and the square plaquette(sp) for the transition, which is specified as $\mathcal{O}(s p)_{1}$, are shown in Fig.1. The suffix 1 corresponds to the assignment of ref. [8] for the number of links $l=4$.

The proper rotations that transform $\mathcal{O}(s p)_{1}$ into itself are $C_{2 z}, C_{4 z}, C_{4 z}^{-1}$ and that transform into $\mathcal{O}(s p)_{1}^{r}$ are $C_{2 x}, C_{2 y}, C_{2 a}$ and $C_{2 b}$.

2. $\mathrm{R}$

The bases and the rectangular plaquette(rp) for the transition which is specified as $\mathcal{O}(r p)_{1}$ are shown in Fig.2

The proper rotation that transforms $\mathcal{O}(r p)_{1}$ into itself is $C_{2 z}$ and that transform into $\mathcal{O}(r p)_{1}^{r}$ are $C_{2 x}$ and $C_{2 y}$.

Here it should be emphasized that, in ref.(3), the R-geometry also contains the four squares with sides $d=1,2,3,4$. These behave as in case LS above. Since the LS-case has more transformation options than the R-case, it is expected that squares generate a larger overall interaction - as is observed in the lattice data.

\section{TR}

The bases and the bent plaquettes(bp) for the transition, which is specified as $\mathcal{O}(b p)_{7}$, are shown in Fig.3. The suffix 7 corresponds to the assignment of [8] for the number of links $l=6$. The symmetry property does not change for larger $l$. 
The proper rotation that transforms $\mathcal{O}(b p)_{7}$ into itself is $C_{2 c}$ and that transform into $\mathcal{O}(b p)_{7}^{r}$ are $C_{2 d}$ and $C_{2 y}$.

4. L

The bases of the linear configuration are shown in Fig.4. The transition now occurs by the insertion of minimum plaquettes twice at the same position. We call it the self-energy operation.

5. Q

The bases and the rectangular plaquette(rp) for the transition which is specified as $\mathcal{O}(r p)_{1}$ are shown in Fig.5. The transition to $B_{2}$ occurs through the self-energy mechanism as in case L.

The proper rotation that transforms $\mathcal{O}(r p)_{1}$ into itself is $C_{2 z}$ and that transform into $\mathcal{O}(r p)_{1}^{r}$ are $C_{2 x}$ and $C_{2 y}$.

6. NP

The bases and the plaquettes( $\mathrm{p}=\mathrm{sp}, \mathrm{rp})$, bent plaquette(bp) and twisted plaquette(tp) for the transition which are specified as $\mathcal{O}(p)_{1} \pm \mathcal{O}(p)_{5}, \mathcal{O}(b p)_{7} \pm \mathcal{O}(b p)_{12}$ and $\mathcal{O}(t p)_{1}$ are shown in Fig.6. The suffices 7 and 12 correspond to the assignment of [8] for $l=6$. The symmetry property does not change for larger $l$. The proper rotations among bases $\mathcal{O}(p)_{1}$ and $\mathcal{O}(p)_{5}$ and those among bases $\mathcal{O}(b p)_{7}$ and $\mathcal{O}(b p)_{12}$ can be obtained by the standard method [9].

The proper rotations that transform $\mathcal{O}(t p)_{1}$ into itself are $C_{3 \beta}, C_{3 \beta}^{-1}$ and that transform into $\mathcal{O}(t p)_{1}^{r}$ are $C_{2 a}, C_{2 d}$ and $C_{2 e}$.

The self-energy mechanism transforms the basis A into the basis $B^{+}$, which is created from A by an insertion of $\mathcal{O}(p)_{1}+\mathcal{O}(p)_{5}$ and $\mathcal{O}(b p)_{7}+\mathcal{O}(b p)_{12}$, but not with $B^{-}$which is created from A by an insertion of $\mathcal{O}(p)_{1}-\mathcal{O}(p)_{5}$ and $\mathcal{O}(b p)_{7}-\mathcal{O}(b p)_{12}$.

\subsection{The gluon overlap factor}

Among the proper rotations that keep the orientation of the plaquette, the operation that transforms to $\mathcal{O}(p)^{r}$, which is related to the charge conjugation, would have the main overlap to the basis $\mathrm{C}$. Typical examples are obtained by transforming the shortest $\bar{q} q$ link in the basis $\underline{B_{2}}$ of the NP geometry and switching the connection of the open circle on the shortest link to make a $q q$ link and a $\bar{q} \bar{q}$ link. Another example can be produced in the 
basis $\underline{B_{3}}$ of NP, but this kind of coupling of the basis $\mathrm{C}$ to the bases $\mathrm{A}$ and $\mathrm{B}$ occurs only in very restricted circumstances: a diagram with three paths from a $q$ or a $\bar{q}$ must be there and the three paths should be colour neutral. Therefore we neglect the rotation that transforms $\mathcal{O}(p)$ to $\mathcal{O}(p)^{r}$.

We define the length of the two paths in the state $\mathrm{A}$ and the distance between the two, in units of the lattice spacing $a$, as $d$ and $r$, respectively. In the case of L, Q and NP, $r$ is defined as the distance between quarks 3 and 4 .

The strong coupling potential in the direct channel is obtained from

$$
<W\left(L, T, g^{2}\right)>=e^{-v(L) T}
$$

which yields $v\left(r_{i j}\right)=b_{s} r_{i j}$. Including the lattice Coulomb interaction and the self-energy $v_{0}$ [2] and defining $v_{i j}$ as (9), we parametrize $v_{A}=v_{13}+v_{24}, v_{B}=$ $v_{14}+v_{23}$.

We fit the gluon overlap factor $f$ for LS, R, TR, L and Q by the condition that the solution $\lambda$ of the equation

$$
\operatorname{det} W=\operatorname{det}\left(\left[\begin{array}{cc}
v_{A} & f v_{A B} \\
f v_{A B} & v_{B}
\end{array}\right]-\lambda\left[\begin{array}{cc}
1 & f / 2 \\
f / 2 & 1
\end{array}\right]\right)=0
$$

agrees with the energy eigenvalues of the lattice calculation 3. It should be added that the parametrization of the two body potential (9) is done for each configuration separately and the deviation of the lattice data from the fitted formula (9) is less than $0.5 \%$.

In the NP case, we need to analyze the lattice calculation data using the bases $\mathrm{A}, B^{+}$and $B^{-}$. We define the overlap factors between $\mathrm{A}$ and $B^{ \pm}$by $f^{ \pm}$. Since

$$
\begin{aligned}
& <O(p)_{i}+O(p)_{j}\left|C_{2 k}\right| O(p)_{i}-O(p)_{j}> \\
= & -<O(p)_{i}\left|C_{2 k}\right| O(p)_{j}>+<O(p)_{j}\left|C_{2 k}\right| O(p)_{i}> \\
= & -<O(p)_{i}-O(p)_{j}\left|C_{2 k}\right| O(p)_{i}+O(p)_{j}>
\end{aligned}
$$

and the matrix $\mathrm{W}$ should be Hermitian, we define the transition matrix between $B^{-}$and $B^{+}$is proportional to the Pauli matrix $-\sigma_{y}$ multiplied by a factor $t$ and choose $f^{ \pm}$to be complex.

We fit $f^{ \pm}$and t from the condition that the solution $\lambda$ 's of the following equation coincide with lowest three energy eigenvalues of the lattice calculation. 


$$
\begin{aligned}
\operatorname{det} W= & \operatorname{det}\left(\left[\begin{array}{ccc}
v_{A} & \sqrt{2} v_{A B} f^{+} & \sqrt{2} v_{A B} f^{-} \\
\sqrt{2} v_{A B} f^{+*} & v_{B} & i t \\
\sqrt{2} v_{A B} f^{-*} & -i t & v_{B}
\end{array}\right]\right. \\
& \left.-\lambda\left[\begin{array}{ccc}
1 & \sqrt{2} f^{+} / 2 & \sqrt{2} f^{-/ 2} \\
\sqrt{2} f^{+*} / 2 & 1 & 0 \\
\sqrt{2} f^{-*} / 2 & 0 & 1
\end{array}\right]\right)=0
\end{aligned}
$$

The matrix elements between $A$ and $B^{ \pm}$are multiplied by $\sqrt{2}$ due to the normalization of the bases of $B^{ \pm}$. Algebraic forms of the three $\lambda$ 's can be obtained by using Mathematica. They are functions of $\left|f^{+}\right|^{2}+\left|f^{-}\right|^{2}$ and $\operatorname{Im}\left(f^{+} f^{-*}\right)$. Therefore $f^{+}$and $f^{-}$are interchangeable.

The explicit forms of $f$ are chosen as follows.

\section{LS}

$$
f=e^{-\left[b_{s} E \mathcal{A}+\sqrt{b_{s}}\left(A_{1}+A_{2}\right) \mathcal{P}\right]}
$$

Here $b_{s}$ is the string tension and $\mathcal{A}=d^{2}$ - the area of the square. The parameter $E$ fixes the overlap of the gluon configuration due to the strong coupling area dependence. Parameters $A_{1}$ and $A_{2}$ fix the overlap due to the links on the perimeter of the area and $\mathcal{P}=$ 4d. $A_{1}$ contains contributions of $C_{2 z}$ and $A_{2}$ contains contributions of $C_{4 z}$ and $C_{4 z}^{-1}$.

In principle, one could include an overall normalization factor $f_{c}$ in $f$. In fact, if one omits the data of $(r, d)=(1,1)$ and fits the overlap factor $f$ with the additional factor $f_{c}$, we obtain a much smaller $\chi^{2}$ with $f_{c} \simeq 0.87$. However, in this case $f$ becomes almost Gaussian and the value of $f$ at $r=0$ and $r=1$ in lattice units becomes very close to each other. This means that when we change the lattice spacing $a$ to a smaller $a^{\prime}$ the slope of $f$ at $(r, d)=(1,1)$ and at $(r, d)=(2,2)$ in the new lattice spacing would be quite different. We compare the data of $\beta=2.4$ which corresponds to $a=0.12 \mathrm{fm}$ and $\beta=2.5$ which corresponds to $a^{\prime}=0.082 \mathrm{fm}$. The parameters of the quark-antiquark potential for the two $\beta$ values are given in Table 1 . The overlap factor $f$ of $(r, d)=(1,1)$ for 
$\beta=2.5$ turns out to be 0.94 . Thus, we fit $f$ as an exponential and not as Gaussian, and fix the normalization to be 1. This means that we incorporate the lattice effect or correction to the area term explicitly into the fitting. We then need to extrapolate the fitted data to the continuum limit, where the perimeter term - except for the self energy term - would become unimportant.

2. $\mathrm{R}$

$$
f=e^{-\left[b_{s} E \mathcal{A}+\sqrt{b_{s}} A_{1} \mathcal{P}\right]}
$$

Here $\mathcal{A}=r d$ and $\mathcal{P}=2(r+d)$. Since $\mathrm{R}$ does not have the symmetry corresonding to $C_{4 z}$ and $C_{4 z}^{-1}$, the $A_{2}$ must be omitted here. Since the area term dominates over the perimeter term as $\mathrm{r}$ and $\mathrm{d}$ becomes large, the difference in the overlap factor in LS and $\mathrm{R}$ becomes small for large plaquettes.

3. TR

$$
f=e^{-\left[b_{s} E \mathcal{A}^{\prime}+\sqrt{b_{s}} A_{3} \mathcal{P}\right]}
$$

We define the projection of the paths 1-4 and 2-3 onto the $\mathrm{x}$ $\mathrm{y}$ plane and onto the $\mathrm{y}-\mathrm{z}$ plane by $(x, z)$ - in lattice units $a$. Therefore the length of the paths in $\mathrm{B}$ is $\sqrt{x^{2}+z^{2}}=r^{\prime}$ and $\mathcal{A}^{\prime}=$ $d r^{\prime}$ is the area of the surface that is bounded by the four links $1-3,3-2,2-4$ and $4-1$. We do not take the sum of the area of the two plaquettes of Fig.3 since for large $d$ and $r$ it would not correspond to the continuum limit of interest. The spectrum for $(d, x, z)=(5,3,4)=(5,4,3)$ becomes very similar to that of LS with $r=d=5$, in this model.

Although a parametrization specific to the cubic symmetry is not desirable, the length of the perimeter is tightly related to the cubic symmetry of the links: i.e. $A_{3}$ contains the contribution of $C_{2 c}$. Thus, we take $\mathcal{P}=2(x+z+d)$ which is the length of the bent plaquette.

4. L

$$
f=e^{-\sqrt{b_{s}} A_{0} 2 r}
$$


In order to simulate the self energy mechanism we take the perimeter of the minimum area along the overlapping links of the basis B. Normalizing $f=1$ for $r=0$, we obtain the above formula.

5. Q

$$
f=\left(e^{-\sqrt{b_{s}} A_{0} 2(r-d)}+e^{-\left[b_{s} E \mathcal{A}^{\prime}+\sqrt{b_{s}} A_{1} \mathcal{P}\right]}\right) / 2
$$

where the first term comes from the same mechanism as the linear (L) and the latter is the rectangular plaquette contribution. The area $\mathcal{A}^{\prime}=r d / 2$ is the area of the triangle 1-3-4, that is half of the plaquette area. $\mathcal{P}=2(r+d)$ is the length of the perimeter of the rectangular plaquette which transforms under $C_{2 z}$.

6. NP

In the nonplanar case, combinations of bent plaquettes $\mathcal{O}(b p)_{i}$ $(i=1, \cdots, 12)$ lead to different parity states 8 . In our restricted plaquette bases the symmetric and antisymmetric combinations of the plaquettes $\mathcal{O}(p)_{1} \pm \mathcal{O}(p)_{5}$ and $\mathcal{O}(b p)_{7} \pm \mathcal{O}(b p)_{12}$ lead to bases $B^{+}$and $B^{-}$respectively.

We parametrize $f^{ \pm}=\left(f_{r e} \pm i f_{i m}\right)$, where

$$
\begin{array}{r}
f_{r e}=\left(e^{-\sqrt{b_{s}} A_{0} 2 r}+2 e^{-\left[b_{s} E \mathcal{A}_{1}+\sqrt{b_{s}} B_{1} \mathcal{P}_{1}\right]}\right. \\
+2 e^{-\left[b_{s} E \mathcal{A}_{2}+\sqrt{b_{s}} B_{2} \mathcal{P}_{2}\right]} \\
\left.+e^{-\left[b_{s} E \mathcal{A}^{\prime \prime}+\sqrt{b_{s}} A_{4} \mathcal{P}_{3}\right]}\right) / 6
\end{array}
$$

and

$$
f_{i m}=\left(e^{-\left[b_{s} E \mathcal{A}_{1}+\sqrt{b_{s}} B_{1} \mathcal{P}_{1}\right]}+e^{-\left[b_{s} E \mathcal{A}_{2}+\sqrt{b_{s}} B_{2} \mathcal{P}_{2}\right]}\right) / 2 .
$$

Here $B_{1}$ in $f_{r e}$ contains the contribution of $C_{2 z} \pm C_{2 y}$, while that in $f_{i m}$ the contribution of $C_{4 x}, C_{4 x}^{-1}$ and $C_{2 e}$. $\mathcal{P}_{1}=2(r+d)$ is the perimeter of the plaquette $\mathcal{O}(p)_{1}$ and $\mathcal{O}(p)_{5}$, and $\mathcal{A}_{1}=$ rd is the corresponding area. $B_{2}$ in $f_{r e}$ contains contributions of $C_{2 c} \pm C_{2 b}$, while that in $f_{i m}$ the contribution of $C_{3 \alpha}, C_{3 \alpha}^{-1}$, and $C_{2 e} . \mathcal{P}_{2}=2(r+2 d)$ is the perimeter of the plaquette $\mathcal{O}(b p)_{7}$ and $\mathcal{O}(p)_{12}$, and $\mathcal{A}_{2}=d(r+d)$ is the corresponding area. $A_{4}$ contains the contribution of $C_{2 c} \pm C_{2 b}$ and $\mathcal{P}_{3}=2(r+2 d)$ is the perimeter of the plaquette $\mathcal{O}(t p)_{1}$. For the corresponding area $\mathcal{A}^{\prime \prime}$ we adopted 
the area of a curved surface

$$
\begin{array}{r}
\mathcal{A}^{\prime \prime}=\int_{0}^{1} d u \int_{0}^{1} d v\left|\left(u \mathbf{r}_{13}+(1-u) \mathbf{r}_{42}\right) \times\left(v \mathbf{r}_{23}+(1-v) \mathbf{r}_{41}\right)\right| \\
=\int_{0}^{1} d u \int_{0}^{1} d v d \sqrt{r^{2}\left(1-2 u+2 u^{2}\right)+d^{2} v^{2}}
\end{array}
$$

which can be calculated analytically.

The ansatz (22) corresponds to an approximation by the regular surface or an approximation of a surface by a sum of straight lines that connect between two flux lines in a 3-d space e.g. 23 and 14 in fig.6. If the four links that surrounds the area are not on a plane, the regular surface is not necessarily the minimal surface. In fact except for the plane surface it is only the helicoidal surface which is minimal and regular 12. A numerical calculation, using the method of [11] shows, however, that the ansatz (22) is a reasonable approximation especially when $r>d$. Since the evaluation of (22) is simple, we adopt this formula for the area of the NP geometry.

The appearance of $t$ in the potential means that the configuration of quarks and anti-quarks are not enough to classify the energy spectrum and we need to specify the gluonic degrees of freedom. It is natural in the flux-tube picture since the links in B are bent and the excited state can be mixed in. The transition potential connects two different symmetry states which we specified by $B^{+}$ and $B^{-}$.

The appearance of perimeter dependent factors and the splitting of $\mathrm{B}$ into $B^{+}$and $B^{-}$come from gluonic degrees of freedom, since the lattice data are not expressed in the bases which contain only the quark degrees of freedom.

\section{$3 \quad$ Numerical results}

In order to fit the parameters $E, A_{i}$ of $\mathrm{LS}, \mathrm{R}, \mathrm{TR}, \mathrm{L}$, and $\mathrm{Q}$, we tried two options;

1. Fit eigen-energies of all the $(r, d)$ sets directly. 
2. Calculate the overlap factor $f_{0}$ from the ground state eigen energy and $f_{1}$ from the first excited state eigen-energy by solving a quadratic equation for a fixed $(r, d)$ set [1] and fit the averages of $f_{0}$ and $f_{1}$ of all the $(r, d)$ sets.

The two options give slightly different results. The error-bars of the eigenenergies at small $(r, d)$ are crucial for the relative magnitudes of the area dependent part and the perimeter dependent part. The error-bar for small $(r, d)$ is smaller than that for large $(r, d)$, but for $(r, d)=(1,1)$, there are problems in fitting the two-body potential $v_{i j}$ as a sum of the three terms given (9)[目]. However, we assume that the lattice effect is well simulated by the perimeter term, and use the small error-bars given by the lattice simulation. The results of the first option i.e. direct eigen-energy fit give following parameters.

1. LS

We obtain $E=0.296(11)$ and $A_{1}+A_{2}=0.080(2)$.

2. $\mathrm{R}$

We fix $E=0.296$ and obtain $A_{1}=0.057(1)$ which means $A_{2}=$ $0.023(3)$.

3. TR

We again fix $E=0.296$ and obtain $A_{3}=-0.091(6)$. In order that the two mesons does not interact for large $r$ it is necessary that $\lim _{r \rightarrow \infty} f=0$. However, the negative value of $A_{3}$ does not cause trouble since the area term dominates in physically reasonable configurations and $f$ tends to 0 for large $r$.

4. L

We obtain $A_{0}=0.197(3)$.

5. Q

We fix $\mathrm{E}=0.296$ and $A_{0}=0.197$ then we find $A_{1}^{\prime}=0.051(2)$ which is close to the value $A_{1}=0.057(1)$ obtained in $\mathrm{R}$. This is a good indication that our parametrization is meaningful. When we use the perimeter $\mathcal{P}=d+r+\sqrt{r^{2}+d^{2}}$ which corresponds to the continuum limit, we obtain for $d=2,3$ and $4, A_{1}^{\prime}=0.0595$ i.e. closer to $A_{1}$. We insist, however, on using the $\mathcal{P}$ that respects the cubic symmetry. 


\section{NP}

In order to fit the parameters $A_{i}$ and $B_{i}$ of the NP geometry we first fit $\left|f^{+}\right|^{2}+\left|f^{-}\right|^{2}=2\left(f_{r e}^{2}+f_{i m}^{2}\right), \operatorname{Im}\left(f^{+} f^{-*}\right)=2 f_{r e} f_{i m}$ and $t$ for each set of $(r, d)$ independently so that the three $\lambda$ coincide with the three lowest eigenenergies. In the case of $d>r+1$ the unperturbed energy of the configuration $\mathrm{B}$ is lower than that of $\mathrm{A}$ and we found difficulty in fitting the data. In these cases one should consider $A^{ \pm}$and $\mathrm{B}$ as the basis, but since the flux tubes of the basis $\mathrm{B}$ are not along the lattice link it is not possible to specify the bases $A^{ \pm}$. We omit these data from the analysis, except $(r, d)=(1,2)$, where the difference in the unperturbed energy is of the order of 0.05 and we can find parameters that fits the data approximately.

We again fixed $E=0.296$ and $A_{0}=0.197$, and searched parameters to fit the $\left|f^{+}\right|^{2}+\left|f^{-}\right|^{2}$ and $\operatorname{Im}\left(f^{+} f^{-*}\right)$ of the data sets of $(r, d)$ that satisfy $r \geq d-1$. We obtained $B_{1}=1.63(72), B_{2}=-0.12(1)$ and $A_{4}=6 \pm 7$. $A_{4}$ consistent with zero means that the coherence of the links on the twisted plaquette is weak. A fit with $A_{4}$ fixed to be zero gives $B_{1}=5.3 \pm 4.7$ and $B_{2}=-0.092(13)$. Although the error-bar of $B_{1}$ is large it is consistent with the previous 1.63(72). The coherence on the bent plaquette parametrized by $B_{2}$ is relatively stable. It is the main term that transforms among different symmetry states. We remark that $B_{2}$ in the NP case and $A_{3}$ in the TR case are both related to the bent plaquette and the magnitudes are similar, while $B_{1}$ and $A_{1}$ are both related to the rectangular plaquette but the magnitude of $B_{1}$ is larger. In the latter case, not all the 4-quarks are sitting on the plaquette $\underline{B_{2}}$ and $\underline{B_{3}}$. These plaquettes can be obtained by multiplying a plaquette to the base $B_{4}$, and by multiplying a plaquette to the base $B_{5}$, respectively, on which all the 4 -quarks are sitting. Since a link in the model bases contains a staple-shaped link, due to the fuzzing, the effective area and the length of the perimeter of the plaquette characterized by the parameter $B_{2}$ would be modified. We cannot, however, estimate the correction and the parameter $B_{1}$ is regarded as a phenomenological parameter. 
The parameter $t$ that connects the bases $B^{+}$and $B^{-}$should tend to 0 as $\mathrm{r}$ and $\mathrm{d}$ increase, and we observed this tendency in the fitting. But for simplicity, we search an average value of the parameter $\mathrm{t}$ that fits the three lowest eigenenergies of all the data of $(r, d)$. Taking $B_{1}=1.63, B_{2}=-0.12$ and $A_{4}=6.19$ and the error-bars of the eigen-energies given by the lattice calculation data, we fitted t. Due to relatively large error-bars in the highest energy state, we sacrifice the accuracy of the fit of the highest state. In fact in the highest state, more complicated configurations could be involved and the fitting with three bases could be a bad approximation. The value we obtained for $t$ is $0.045(10)$.

\section{Discussion and Conclusion}

We analyzed the four-quark energies in SU(2) calculated by lattice Monte Carlo in a model which is inspired by the resonating group calculation of hadron interactions. The transition matrix element between different bases $\mathrm{A}$ and $\mathrm{B}$ is assumed to be proportional to the gluon overlap factor and this factor is then parametrized by a product of an area dependent and a perimeter dependent term. The perimeter dependent term was introduced by considering transition between degenerate $Z_{2}$ symmetric bases which in the large lattice or in the continuum limit would vanish except the self energy term. In the case of large square(LS), rectangular(R), tilted rectangular(TR), linear $(\mathrm{L})$ and quadrilateral(Q) configurations, we found reasonable parameters for the perimeter dependent term which are characterized by the cubic symmetry. In the non-planar(NP) case, we observed that it is necessary to take into acount the gluonic degrees of freedom explicitly. The base B was split into two hybrid configurations: $B^{+}$and $B^{-}$. Using $A$ and $B^{ \pm}$as the bases, we parametrized the gluon overlap factor for the NP.

We treated, the error-bars given by the lattice Monte Carlo as meaningful also for the small plaquettes and checked whether a consistent parametrization for different geometries is possible. We simulated the deviation from an area dependence by adding a perimeter dependent contribution.

The parameters for the perimeter dependent part are $A_{0}, A_{1}, A_{2}, A_{3}, A_{4}, B_{1}$ and $B_{2} . A_{1}^{\prime}$ in the $\mathrm{Q}$ case is consistent with that of $\mathrm{R}$ case, and $B_{2}$ in the NP case is consistent with $A_{3}$ in TR case. These coincidences are not trivial 
and are encouraging for any further analysis based on the cubic symmetry of the flux tube geometry. $A_{4}$ is consistent with 0 and we could well omit it. Therefore, there are 5 parameters for the perimeter terms and one parameter for the area to fit the lattice Monte Carlo energy spectra.

We expect that in the continuum limit $f$ is completely given by the area term except for the self energy contribution. A comparison of the data at $\beta=2.4$ and $\beta=2.5$ for the LS configuration suggests that the parameters of the perimeter term in fact decrease as the lattice constant $a$ becomes small. Since the Monte Carlo data contain lattice artefacts, in order to analyze the data for a specific configuration we need to choose proper bases so that these artefacts are eliminated. Since the artefacts are represented by the perimeter term, its cubic symmetry property is essential for choosing the proper bases.

S.F thanks the Academy of Finland and the Japan Society for the Promotion of Science for the support of the research from February 7 to April 8 of 1994. He is also grateful to the Research Institute for Theoretical Physics of the University of Helsinki for their kind hospitality. 


\section{References}

[1] A.M.Green, C.Michael and J.E.Paton, Nucl. Phys.A554 (1993) 701.

[2] A.M.Green, C.Michael, J.E.Paton and M.E.Sainio, Int. J. Mod. Phys. E2 (1993) 479.

[3] A.M.Green, C.Michael and M.Sainio, preprint HU-TFT-94-7 (1994)

[4] S.Perantonis, A.Huntley and C.Michael, Nucl.Phys.B326 (1989) 544

[5] H.Matsuoka and D.Sivers, Phys. Rev. D33 (1986) 1441

[6] A.M.Green, G.Q.Liu and S.Wycech, Nucl.Phys.A509 (1989) 529.

[7] M.Creutz, Quarks, gluons and lattices, Cambridge University Press, Cambridge, 1983, p.103

[8] B.Berg and A.Billoire, Nucl. Phys.B221 (1983) 109

[9] J.F.Cornwell, Group Theory in Physics, vol 1, Academic Press, London, 1984.

[10] Yu.M.Makeenko and A.A. Migdal, Phys. Lett. 97B (1980) 253.

[11] W.Businger et al., The Mathematica Journal 4 (1994) 70.

[12] M.P. do Carmo, Differential Geometry of Curves and Surfaces, (Prentice-Hall New Jersey), 1976, Differntialgeometrie von Kurven und Flächen, (Vieweg, Braunschweig), 1983, p.149 . 
Figure 1: The basic four-quark geometries of Large square(LS). $\underline{A}$ is the configuration of the base A. $\underline{B}_{i}$ are the configurations of the base B. The linked $q \bar{q}$ of the bases $\mathrm{A}$ are assigned 1-3 and 2-4, and those of the bases B are 1-4 and 2-3.

Figure 2: Same as Fig.1 but for Rectangle(R).

Figure 3: Same as Fig.1 but for Tilted rectangle(TR).

Figure 4: Same as Fig.1 but for Linear(L).

Figure 5: Same as Fig.1 but for Quadrilateral(Q).

Figure 6: Same as Fig.1 but for Non-planar(NP).

Table 1: $b_{s}, e$ and $v_{0}$ of the quark-antiquark potential

\begin{tabular}{|r|r|r|r|}
\hline$\beta$ & $b_{s}$ & $\mathrm{e}$ & $v_{0}$ \\
\hline 2.4 & 0.07169 & 0.245 & 0.550 \\
2.5 & 0.03781 & 0.215 & 0.523 \\
\hline
\end{tabular}


This figure "fig1-1.png" is available in "png" format from: http://arxiv.org/ps/hep-lat/9409006v1 